\title{
Pair production of the heavy leptons associated with a gauge boson $\gamma$ or $Z$ at the ILC
}

\author{
Qing-Guo Zeng, Chong-Xing Yue,* and Jiao Zhang \\ Department of Physics, \\ Liaoning Normal University, \\ Dalian 116029, China
}

\begin{abstract}
The T-odd leptons are the typical particles predicted by the littlest Higgs model with T-parity (LHT model) and the observation of these particles might be regarded as the direct evidence of the LHT model. In this paper, we investigate the production of a pair of T-odd leptons associated with a gauge boson $V(\gamma$ or $Z)$ at the international linear $e^{+} e^{-}$collider (ILC). The numerical results show that the possible signals of the T-odd leptons may be detected in the future ILC experiments.
\end{abstract}

PACS numbers: 14.60.Hi, 12.60.-i, 13.66.De

*Electronic address: cxyue@lnnu.edu.cn 


\section{INTRODUCTION}

The standard model (SM) provides an excellent effective field theory description of almost all particle physics experiments. However, the theoretical shortcomings of the SM, such as quadratic divergencies, the triviality of a $\phi^{4}$ theory, etc, suggest that it should be embedded in a larger scheme. Many popular new physics (NP) models beyond the SM have been proposed, and some of which predict the existence of new charged leptons. Any signal for such kind of particles in future high energy experiments will play a milestone role in discovery of NP. Thus, studying production and decay of the new charged leptons in future high energy collider experiments is of special interest.

Little Higgs theory [1] is proposed as an interesting solution to the so-called hierarchy problem of the SM and can be regarded as one of the important candidates for NP beyond the SM. Among of the little Higgs models, the littlest Higgs (LH) model [2] has all essential features of the little Higgs models. However, the original version of the LH model suffers from precision electroweak (EW) constrains, the NP effects are small as the NP scale $f$ is required to be above $2-3 \mathrm{TeV}$ in order to satisfy the $\mathrm{EW}$ precision constraints, which re-introduces the fine tuning and the little hierarchy problem [2] [3]. The LH model with T-parity (LHT) [4] [5] [6] is one of the attractive little Higgs models. In the LHT model, all dangerous tree-level contributions to low energy EW observables are forbidden by Tparity and hence the corrections to low energy EW observables are loop-suppressed and

small [4] [7]. As a result, the relatively low new particle mass scale $f$ is still allowed by data, e.g., $f>500 G e V$ [7].

In the LHT model, particle fields are divided into T-even and T-odd sectors under T-parity and the SM fields are T-even. In order to implement T-parity in the fermion sector, one introduces three doublets of mirror quarks and mirror leptons, which have T-odd parity, transform vectorially under $S U(2)_{L}$ and can be given large masses. These mirror fermions have new flavor violating interactions with the SM fermions mediated by the new gauge bosons and at higher order by the triplet scalar, which might generate significantly contributions to some flavor violation processes [6] [7] [8] [9]. It has been shown that the LHT mirror fermion interactions can yield large NP effects in the quark sector [6] [7] [9] and the lepton sector [10] [11] [12].

So far, lots of studies about the heavy charged leptons have previously been done at 
hadron colliders. However, there exists some difficulties to detect heavy lepton production at the CERN large hadron collider (LHC), due to large backgrounds. Compared to hadron colliders, the future international linear $e^{+} e^{-}$collider (ILC) has the advantage in performing experimental measurement with a particularly clean environment [13]. Furthermore, ILC can provide complementary information for NP with performing precision measurements that would complete the LHC results. Many works involving the new charged leptons have been given at the ILC. Studies about the heavy charged leptons predicted by the LHT model have previously been done at the LHC [14] and ILC[15]. As a complementary production mode to the former research, this paper is to study the production processes of the T-odd leptons in association with a neutral gauge boson $V(=\gamma$, or $Z$ ) at the ILC experiments.

This paper is organized as follows. In section II, we give a brief review of the LHT model and then give the relevant couplings. In section III, we devote to the computation of the production cross section of the process $e^{+} e^{-} \rightarrow \gamma \bar{L}_{i} L_{j}$. The study of the production process $e^{+} e^{-} \rightarrow Z \bar{L}_{i} L_{j}$ is presented in section IV. Some phenomenological analysis are included in the above two sections. The conclusions are given in section $\mathrm{V}$.

\section{A BRIEF REVIEW OF THE LHT MODEL}

The LHT model is based on an $S U(5) / S O(5)$ global symmetry breaking pattern. A subgroup $[S U(2) \times U(1)]_{1} \times[S U(2) \times U(1)]_{2}$ of the $S U(5)$ global symmetry is gauged, and at the scale $f$ it is broken into the SM EW symmetry $S U(2)_{L} \times U(1)_{Y}$. T-parity is an automorphism that exchanges the $[S U(2) \times U(1)]_{1}$ and $[S U(2) \times U(1)]_{2}$ gauge symmetries. The T-even combinations of the gauge fields are the EW gauge bosons, and the T-odd combinations are their T-parity partners. After taking into account EW symmetry breaking, at the order of $\nu^{2} / f^{2}$, the masses of the T-odd set of the $S U(2) \times U(1)$ gauge bosons are given by:

$$
M_{Z_{H}}=M_{W_{H}}=g f\left(1-\frac{\nu^{2}}{8 f^{2}}\right), \quad M_{B_{H}}=\frac{g^{\prime} f}{\sqrt{5}}\left(1-\frac{5 \nu^{2}}{8 f^{2}}\right) .
$$

Where $g$ and $g^{\prime}$ are the corresponding coupling constants of $S U(2)_{L}$ and $U(1)_{Y} \cdot \nu=$ $246 \mathrm{GeV}$ is the EW scale and $f$ is the scale parameter of the gauge symmetry breaking of the LHT model. Moreover, because of the smallness of $g^{\prime}$, the T-odd gauge boson $B_{H}$ 
is the lightest T-odd particle, which is stable, electrically neutral, and weakly interacting particle. Thus, it can be seen as an attractive dark matter candidate [16]. To avoid severe constraints and simultaneously implement T-parity, one needs to double the SM fermion doublet spectrum [4] [5]. The T-even combination is associated with the $S U(2)_{L}$ doublet, while the T-odd combination is its T-parity partner. At the leading order, the masses of the T-odd fermions can be written in a unified manner as:

$$
M_{F_{H}^{i}}=\sqrt{2} \kappa_{i} f
$$

where the Yukawa couplings $\kappa_{i}$ can in general depend on the fermion species $i$.

One of the important ingredients of the mirror sector in the LHT model is the existence of CKM-like unitary mixing matrices. Mirror fermions are characterized by new flavor interactions with SM fermions and heavy gauge bosons, which involve two new unitary mixing matrices in the quark sector, $V_{H u}$ and $V_{H d}$, and two in the lepton sector, $V_{H l}$ and $V_{H \nu}$ [7] [8]. These mirror mixing matrices parameterize flavor-changing (FC) interactions between the SM fermions and the mirror fermions. The two CKM-like unitary mixing matrices $V_{H l}$ and $V_{H \nu}$ satisfy the following physical constrains:

$$
V_{H \nu}^{\dagger} V_{H l}=V_{P M N S}
$$

Here the Pontecorvo-Maki-Nakagata-Saki (PMNS) matrix $V_{P M N S}$ is defined through neutrino mixing. $V_{H l}$, the most important mixing matrix in the present paper, parameterizes the interactions of light charged leptons with mirror neutrinos, mediated by $W_{H}^{ \pm}$, and with mirror charged leptons, mediated by $Z_{H}$ and $B_{H}$. On the other hand, $V_{H \nu}$ parameterizes the interactions of light neutrinos with mirror leptons. Ref. [6] parameterizes $V_{H l}$ with three mixing angles $\theta_{12}^{l}, \theta_{23}^{l}, \theta_{13}^{l}$ and three complex phases $\delta_{12}^{l}, \delta_{23}^{l}, \delta_{13}^{l}$ :

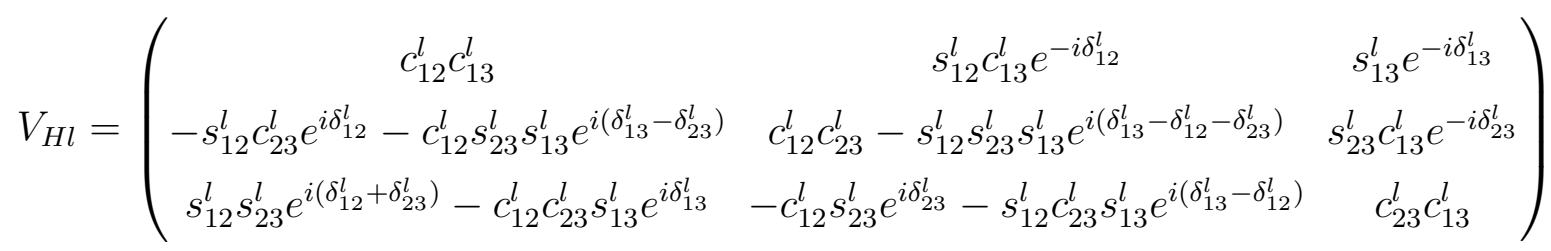

For the matrix $V_{P M N S}$, we take the standard parameterization form with parameters given by the neutrino experiments [17]. As no constraints on the PMNS phases exist, we will set the three Majorana phases of $V_{P M N S}$ to zero in our numerical estimations. 
The Feynman rules of the T-odd leptons (mirror leptons) which are related to our calculation can be written as [6]:

$$
\begin{gathered}
\gamma \bar{L}_{i} L_{j}:-i e \gamma^{\mu} \delta_{i j}, \quad B_{H} \bar{L}_{i} l_{j}: \frac{i e}{C_{W}}\left[\frac{1}{10}+\frac{5 C_{W}^{2}}{8\left(5 C_{W}^{2}-S_{W}^{2}\right)} \frac{v^{2}}{f^{2}}\right]\left(V_{H l}\right)_{i j} \gamma^{\mu} P_{L} ; \\
Z \bar{L}_{i} L_{j}: \frac{i e}{S_{W} C_{W}}\left[-\frac{1}{2}+S_{W}^{2}\right] \gamma^{\mu} \delta_{i j} ; \quad Z_{H} \bar{L}_{i} l_{j}: \frac{i e}{S_{W}}\left[-\frac{1}{2}+\frac{S_{W}^{2}}{8\left(5 C_{W}^{2}-S_{W}^{2}\right)} \frac{v^{2}}{f^{2}}\right]\left(V_{H l}\right)_{i j} \gamma^{\mu} P_{L}
\end{gathered}
$$

where $P_{L}=\frac{1}{2}\left(1-\gamma_{5}\right)$ is the left-handed projection operator. $S_{W}$ represents the $\sin \theta_{W}$ of the Weinberg angle $\theta_{W} . l_{i}$ and $L_{j}$ represent the three family leptons $e, \mu$, and $\tau$ and the three family T-odd leptons, respectively.

Certainly, the trilinear coupling $Z_{H} B_{H} Z$ can also contribute to the process $e^{+} e^{-} \rightarrow$ $V \bar{L}_{i} L_{j}$. However, this kind of couplings are induced at the one-loop level by a fermion triangle and its contributions are very small [18]. Thus, in our following calculation, we will neglect the contributions of this kind of couplings .

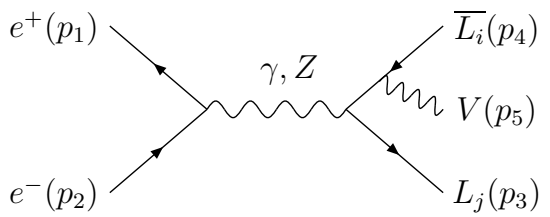

(a)

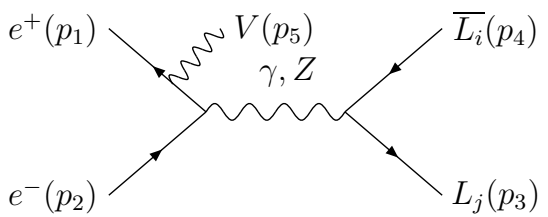

(c)

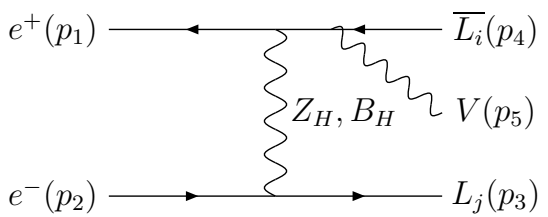

(e)

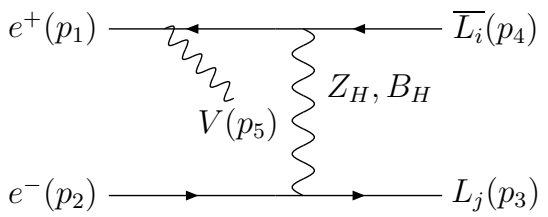

(g)

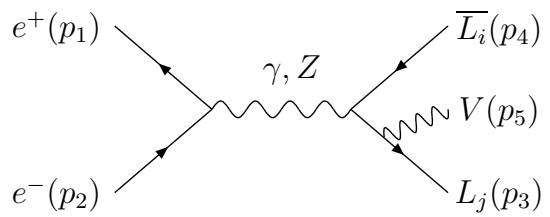

(b)

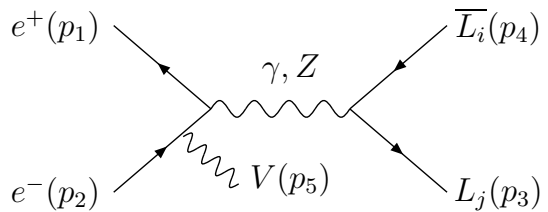

(d)

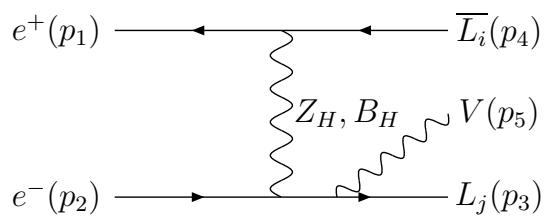

(f)

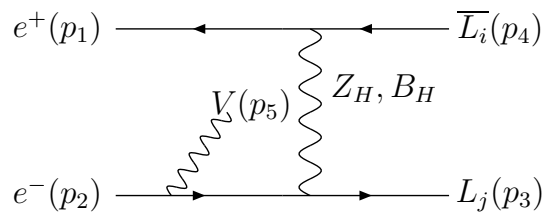

(h)

FIG. 1: The Feynman diagrams of the process $e^{+} e^{-} \rightarrow V \bar{L}_{i} L_{j}$ in the LHT model. 


\section{THE PROCESSES $e^{+} e^{-} \rightarrow \gamma \bar{L}_{i} L_{j}$}

With the above couplings, the Feynman diagrams for the process $e^{+}\left(p_{1}\right) e^{-}\left(p_{2}\right) \rightarrow$ $\gamma\left(p_{5}\right) \bar{L}_{i}\left(p_{4}\right) L_{j}\left(p_{3}\right)$ are shown in Fig.1. The production amplitude can be written as

$$
\begin{aligned}
M_{1}= & M_{a}^{\gamma \gamma}+M_{a}^{Z \gamma}+M_{b}^{\gamma \gamma}+M_{b}^{Z \gamma}+M_{c}^{\gamma \gamma}+M_{c}^{Z \gamma}+M_{d}^{\gamma \gamma}+M_{d}^{Z \gamma}+M_{e}^{B_{H} \gamma} \\
& +M_{e}^{Z_{H} \gamma}+M_{f}^{B_{H} \gamma}+M_{f}^{Z_{H} \gamma}+M_{g}^{B_{H} \gamma}+M_{g}^{Z_{H} \gamma}+M_{h}^{B_{H} \gamma}+M_{h}^{Z_{H} \gamma}
\end{aligned}
$$

with

$$
\begin{aligned}
& M_{a}^{\gamma \gamma}=-i e^{3} G\left(p_{1}+p_{2}, 0\right) G\left(p_{4}+p_{5}, M_{L}\right) \bar{v}\left(p_{1}\right) \gamma^{\mu} u\left(p_{2}\right) \bar{u}\left(p_{3}\right) \gamma_{\mu}\left[-\left(\not p_{4}+\not p_{5}\right)+M_{L}\right] \\
& \times \notin\left(p_{5}\right) v\left(p_{4}\right), \\
& M_{a}^{Z \gamma}=\frac{-i e^{3}}{S_{W}^{2} C_{W}^{2}}\left(-\frac{1}{2}+S_{W}^{2}\right) G\left(p_{1}+p_{2}, M_{Z}\right) G\left(p_{4}+p_{5}, M_{L}\right) \bar{v}\left(p_{1}\right) \gamma^{\mu}\left[\left(-\frac{1}{2}+S_{W}^{2}\right) P_{L}\right. \\
& \left.+\left(S_{W}^{2}\right) P_{R}\right] u\left(p_{2}\right) \bar{u}\left(p_{3}\right) \gamma_{\mu}\left[-\left(\not p_{4}+\not p_{5}\right)+M_{L}\right] \notin\left(p_{5}\right) v\left(p_{4}\right), \\
& M_{b}^{\gamma \gamma}=-i e^{3} G\left(p_{1}+p_{2}, 0\right) G\left(p_{3}+p_{5}, M_{L}\right) \bar{v}\left(p_{1}\right) \gamma^{\mu} u\left(p_{2}\right) \bar{u}\left(p_{3}\right) \notin\left(p_{5}\right)\left[\not p_{3}+\not p_{5}+M_{L}\right] \\
& \times \gamma_{\mu} v\left(p_{4}\right), \\
& M_{b}^{Z \gamma}=\frac{-i e^{3}}{S_{W}^{2} C_{W}^{2}}\left(-\frac{1}{2}+S_{W}^{2}\right) G\left(p_{1}+p_{2}, M_{Z}\right) G\left(p_{3}+p_{5}, M_{L}\right) \bar{v}\left(p_{1}\right) \gamma^{\mu}\left[\left(-\frac{1}{2}+S_{W}^{2}\right) P_{L}\right. \\
& \left.+\left(S_{W}^{2}\right) P_{R}\right] u\left(p_{2}\right) \bar{u}\left(p_{3}\right) \notin\left(p_{5}\right)\left[\not p_{3}+\not p_{5}+M_{L}\right] \gamma_{\mu} v\left(p_{4}\right), \\
& M_{c}^{\gamma \gamma}=-i e^{3} G\left(p_{1}-p_{5}, M_{e}\right) G\left(p_{3}+p_{4}, 0\right) \bar{v}\left(p_{1}\right) \notin\left(p_{5}\right)\left[-\left(\not p_{1}-\not p_{5}\right)+M_{e}\right] \gamma^{\mu} u\left(p_{2}\right) \bar{u}\left(p_{3}\right) \\
& \times \gamma_{\mu} v\left(p_{4}\right), \\
& M_{c}^{Z \gamma}=\frac{-i e^{3}}{S_{W}^{2} C_{W}^{2}}\left(-\frac{1}{2}+S_{W}^{2}\right) G\left(p_{1}-p_{5}, M_{e}\right) G\left(p_{3}+p_{4}, M_{Z}\right) \bar{v}\left(p_{1}\right) \notin\left(p_{5}\right)\left[-\left(\not p_{1}-\not p_{5}\right)\right. \\
& \left.+M_{e}\right] \gamma^{\mu}\left[\left(-\frac{1}{2}+S_{W}^{2}\right) P_{L}+\left(S_{W}^{2}\right) P_{R}\right] u\left(p_{2}\right) \bar{u}\left(p_{3}\right) \gamma_{\mu} v\left(p_{4}\right), \\
& M_{d}^{\gamma \gamma}=-i e^{3} G\left(p_{2}-p_{5}, M_{e}\right) G\left(p_{3}+p_{4}, 0\right) \bar{v}\left(p_{1}\right) \gamma^{\mu}\left[\left(\not p_{2}-\not p_{5}\right)+M_{e}\right] \notin\left(p_{5}\right) u\left(p_{2}\right) \bar{u}\left(p_{3}\right) \\
& \times \gamma_{\mu} v\left(p_{4}\right) \\
& M_{d}^{Z \gamma}=\frac{-i e^{3}}{S_{W}^{2} C_{W}^{2}}\left(-\frac{1}{2}+S_{W}^{2}\right) G\left(p_{2}-p_{5}, M_{e}\right) G\left(p_{3}+p_{4}, M_{Z}\right) \bar{v}\left(p_{1}\right) \gamma^{\mu}\left[\left(-\frac{1}{2}+S_{W}^{2}\right) P_{L}\right. \\
& \left.+\left(S_{W}^{2}\right) P_{R}\right]\left[\left(\not p_{2}-\not p_{5}\right)+M_{e}\right] \notin\left(p_{5}\right) u\left(p_{2}\right) \bar{u}\left(p_{3}\right) \gamma_{\mu} v\left(p_{4}\right),
\end{aligned}
$$




$$
\begin{aligned}
& M_{e}^{B_{H} \gamma}=\frac{-i e^{3}}{C_{W}^{2}}\left[\frac{1}{10}+\frac{5 C_{W}^{2}}{8\left(5 C_{W}^{2}-S_{W}^{2}\right)} \frac{\nu^{2}}{f^{2}}\right]^{2}\left(V_{H l}\right)_{i e}\left(V_{H l}\right)_{e j} G\left(p_{4}+p_{5}, M_{L}\right) G\left(p_{2}-p_{3}, M_{B_{H}}\right) \\
& \times \bar{v}\left(p_{1}\right) \gamma^{\mu} P_{L}\left[-\left(\not p_{4}+\not p_{5}\right)+M_{L}\right] \notin\left(p_{5}\right) v\left(p_{4}\right) \bar{u}\left(p_{3}\right) \gamma_{\mu} P_{L} u\left(p_{2}\right), \\
& M_{e}^{Z_{H} \gamma}=\frac{-i e^{3}}{S_{W}^{2}}\left[-\frac{1}{2}+\frac{S_{W}^{2}}{8\left(5 C_{W}^{2}-S_{W}^{2}\right)} \frac{\nu^{2}}{f^{2}}\right]^{2}\left(V_{H l}\right)_{i e}\left(V_{H l}\right)_{e j} G\left(p_{4}+p_{5}, M_{L}\right) G\left(p_{2}-p_{3}, M_{Z_{H}}\right)
\end{aligned}
$$

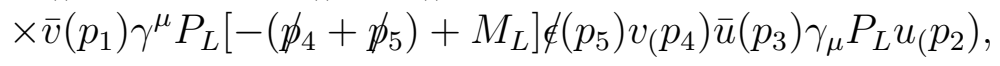

$$
\begin{aligned}
& M_{f}^{B_{H} \gamma}=\frac{-i e^{3}}{C_{W}^{2}}\left[\frac{1}{10}+\frac{5 C_{W}^{2}}{8\left(5 C_{W}^{2}-S_{W}^{2}\right)} \frac{\nu^{2}}{f^{2}}\right]^{2}\left(V_{H l}\right)_{i e}\left(V_{H l}\right)_{e j} G\left(p_{4}-p_{1}, M_{B_{H}}\right) G\left(p_{3}+p_{5}, M_{L}\right) \\
& \times \bar{v}\left(p_{1}\right) \gamma^{\mu} P_{L} v\left(p_{4}\right) \bar{u}\left(p_{3}\right) \notin\left(p_{5}\right)\left[p_{3}+\not p_{5}+M_{L}\right] \gamma_{\mu} P_{L} u\left(p_{2}\right), \\
& M_{f}^{Z_{H} \gamma}=\frac{-i e^{3}}{S_{W}^{2}}\left[-\frac{1}{2}+\frac{S_{W}^{2}}{8\left(5 C_{W}^{2}-S_{W}^{2}\right)} \frac{\nu^{2}}{f^{2}}\right]^{2}\left(V_{H l}\right)_{i e}\left(V_{H l}\right)_{e j} G\left(p_{4}-p_{1}, M_{Z_{H}}\right) G\left(p_{3}+p_{5}, M_{L}\right) \\
& \times \bar{v}\left(p_{1}\right) \gamma^{\mu} P_{L} v\left(p_{4}\right) \bar{u}\left(p_{3}\right) \notin\left(p_{5}\right)\left[p_{3}+\not p_{5}+M_{L}\right] \gamma_{\mu} P_{L} u\left(p_{2}\right) \text {, } \\
& M_{g}^{B_{H} \gamma}=\frac{-i e^{3}}{C_{W}^{2}}\left[\frac{1}{10}+\frac{5 C_{W}^{2}}{8\left(5 C_{W}^{2}-S_{W}^{2}\right)} \frac{\nu^{2}}{f^{2}}\right]^{2}\left(V_{H l}\right)_{i e}\left(V_{H l}\right)_{e j} G\left(p_{5}-p_{1}, M_{e}\right) G\left(p_{2}-p_{3}, M_{B_{H}}\right) \\
& \times \bar{v}\left(p_{1}\right) \notin\left(p_{5}\right)\left[\not p_{5}-\not p_{1}+M_{e}\right] \gamma^{\mu} P_{L} v\left(p_{4}\right) \bar{u}\left(p_{3}\right) \gamma_{\mu} P_{L} u\left(p_{2}\right) \text {, } \\
& M_{g}^{Z_{H} \gamma}=\frac{-i e^{3}}{S_{W}^{2}}\left[-\frac{1}{2}+\frac{S_{W}^{2}}{8\left(5 C_{W}^{2}-S_{W}^{2}\right)} \frac{\nu^{2}}{f^{2}}\right]^{2}\left(V_{H l}\right)_{i e}\left(V_{H l}\right)_{e j} G\left(p_{5}-p_{1}, M_{e}\right) G\left(p_{2}-p_{3}, M_{Z_{H}}\right) \\
& \times \bar{v}\left(p_{1}\right) \notin\left(p_{5}\right)\left[p_{5}-\not p_{1}+M_{e}\right] \gamma^{\mu} P_{L} v\left(p_{4}\right) \bar{u}\left(p_{3}\right) \gamma_{\mu} P_{L} u\left(p_{2}\right), \\
& M_{h}^{B_{H} \gamma}=\frac{-i e^{3}}{C_{W}^{2}}\left[\frac{1}{10}+\frac{5 C_{W}^{2}}{8\left(5 C_{W}^{2}-S_{W}^{2}\right)} \frac{\nu^{2}}{f^{2}}\right]^{2}\left(V_{H l}\right)_{i e}\left(V_{H l}\right)_{e j} G\left(p_{4}-p_{1}, M_{B_{H}}\right) G\left(p_{2}-p_{5}, M_{e}\right) \\
& \times \bar{v}\left(p_{1}\right) \gamma^{\mu} P_{L} v\left(p_{4}\right) \bar{u}\left(p_{3}\right) \gamma_{\mu} P_{L}\left[\not p_{2}-\not p_{5}+M_{e}\right] \notin\left(p_{5}\right) u\left(p_{2}\right) \text {, } \\
& M_{h}^{Z_{H} \gamma}=\frac{-i e^{3}}{S_{W}^{2}}\left[-\frac{1}{2}+\frac{S_{W}^{2}}{8\left(5 C_{W}^{2}-S_{W}^{2}\right)} \frac{\nu^{2}}{f^{2}}\right]^{2}\left(V_{H l}\right)_{i e}\left(V_{H l}\right)_{e j} G\left(p_{4}-p_{1}, M_{Z_{H}}\right) G\left(p_{2}-p_{5}, M_{e}\right) \\
& \times \bar{v}\left(p_{1}\right) \gamma^{\mu} P_{L} v\left(p_{4}\right) \bar{u}\left(p_{3}\right) \gamma_{\mu} P_{L}\left[p_{2}-\not p_{5}+M_{e}\right] \notin\left(p_{5}\right) u\left(p_{2}\right) .
\end{aligned}
$$

Where $G(p, M)=\frac{1}{p^{2}-M^{2}}$ denotes the propagator of the particle. $p_{1}$ and $p_{2}$ refer to the incoming momentum of the incoming $e^{+}$and $e^{-}$, respectively. $p_{4}, p_{3}$ and $p_{5}$ are the momenta of the outgoing final states $\bar{L}_{i}, L_{j}$ and $\gamma$.

With the above production amplitudes, the production cross section can be directly obtained. In the calculation of the cross section, instead of calculating the square of the amplitudes analytically, we calculate the amplitudes numerically by using the method 
of [19], which can simplify our calculation. In our following calculation, the SM input parameters are taken as $S_{W}^{2}=0.231, m_{Z}=91.187 \mathrm{GeV}$ and the fine-structure constant $\alpha=1 / 128[20]$.

From above discussions, we can see that the production cross sections $\sigma\left(\gamma \bar{L}_{i} L_{j}\right)$ for the processes $e^{+} e^{-} \rightarrow \gamma \bar{L}_{i} L_{j}$ are dependent on the model-dependent free parameters, the symmetry breaking scale $f$, the mirror lepton masses $M_{L_{i}}$, and the matrix elements $\left(V_{H l}\right)_{i j}$. The matrix elements $\left(V_{H l}\right)_{i j}$ can be determined through $V_{H l}=V_{H \nu} V_{P M N S}$. In order to simply the calculation and avoid any additional parameters, we take $V_{H l}=V_{P M N S}$, which means that the T-odd leptons have no impact on the flavor violating observable in the neutrino sector. For the matrix $V_{P M N S}$, the standard parameterization form with parameters given by the neutrino experiments [17]. References [10] [11] have shown that, for $V_{H l}=V_{P M N S}$, to make the $\mu \rightarrow e \gamma$ and $\mu^{-} \rightarrow e^{-} e^{+} e^{-}$decay rates consistent with the present experimental upper bounds, the spectrum of the T-odd leptons (mirror leptons) must be quasi-degenerate. So we will fix the mirror lepton masses $M_{L e}=M_{L \mu}=M_{L \tau}=$ $M_{L}$, and take the symmetry breaking scale $f$ and the mirror lepton mass $M_{L}$ as free parameters. Furthermore, in order to make our numerical results more realistic, we will apply the cut on the transverse momentum for radiated photon as $P_{T}^{\gamma}>P_{T, \text { cut }}^{\gamma}$ with $P_{T, \text { cut }}^{\gamma}=15 \mathrm{GeV}$.

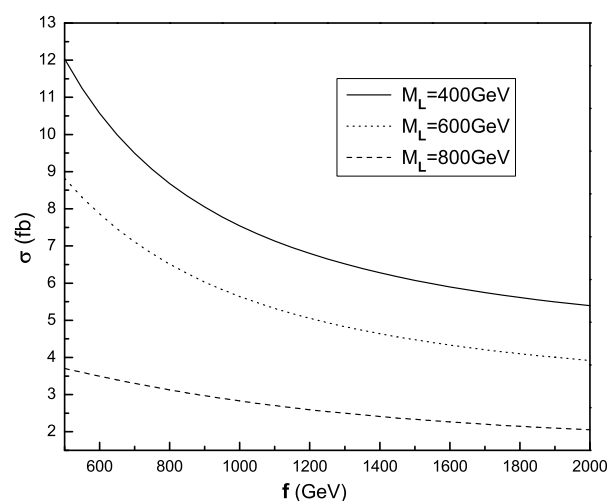

(a)

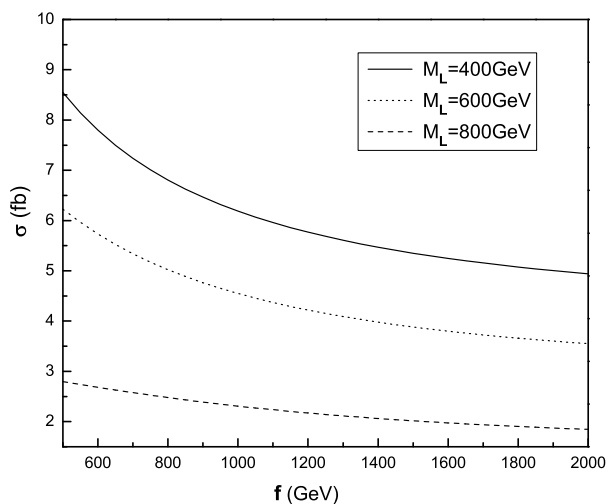

(b)

FIG. 2: The production cross sections (a) $\sigma\left(\gamma \bar{L}_{e} L_{\mu}\right)$ and (b) $\sigma\left(\gamma \bar{L}_{\mu} L_{\mu}\right)$ as function of the scale parameter $f$ for $\sqrt{s}=2 T e V$ and three values of the T-odd lepton mass $M_{L}$. 
From Ref.[17] we can find that the values of the matrix elements $\left(V_{P M N S}\right)_{e \tau}$ and $\left(V_{P M N S}\right)_{\tau e}$ are smaller than those of $\left(V_{P M N S}\right)_{e \mu}$ and $\left(V_{P M N S}\right)_{\mu e}$, respectively. Therefore, it can be speculated that the production cross sections $\sigma\left(\gamma \bar{L}_{\tau} L_{\tau}\right)$ and $\sigma\left(\gamma \bar{L}_{e} L_{\tau}\right)$ [ or $\left.\sigma\left(\gamma \bar{L}_{\mu} L_{\tau}\right)\right]$ are smaller than $\sigma\left(\gamma \bar{L}_{\mu} L_{\mu}\right)$ and $\sigma\left(\gamma \bar{L}_{e} L_{\mu}\right)$, respectively. So we only give the cross sections $\sigma\left(\gamma \bar{L}_{\mu} L_{\mu}\right)$ and $\sigma\left(\gamma \bar{L}_{e} L_{\mu}\right)$ in the following calculation. The PMNS matrix $V_{P M N S}$ have been constructed in Ref.[17] based on PDG parametrization and the available data from oscillation experiments. To simply the numerical results, we take $\left(V_{P M N S}\right)_{e e}=0.82,\left(V_{P M N S}\right)_{\mu e}=0.50$ and $\left(V_{P M N S}\right)_{e \mu}=0.55$.

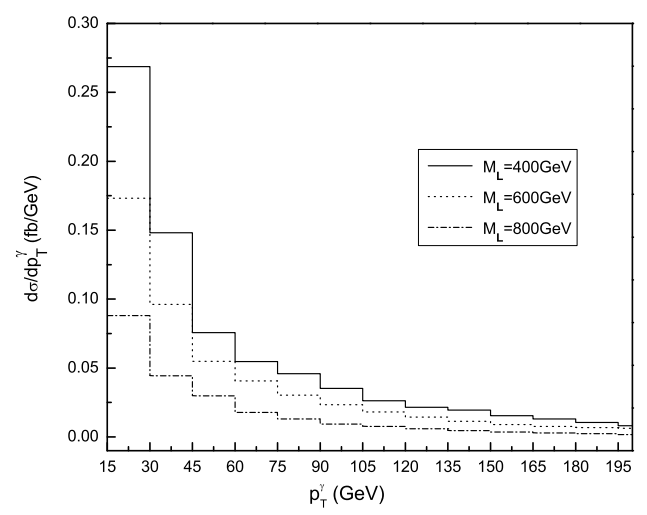

(a)

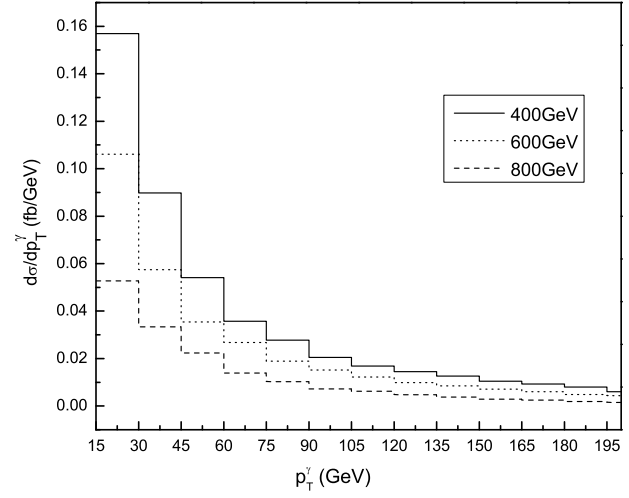

(b)

FIG. 3: The distributions of the transverse momentum of $\gamma$ photon $P_{T}^{\gamma}$ for the $e^{+} e^{-} \rightarrow \gamma \bar{L}_{e} L_{\mu}$ process with (a) $f=0.5 \mathrm{TeV}$, (b) $f=1 \mathrm{TeV}$ for $\sqrt{s}=2 \mathrm{TeV}$ and three values of the T-odd lepton mass $M_{L}$.

In Fig.2, we plot the cross sections $\sigma\left(\gamma \bar{L}_{\mu} L_{\mu}\right)$ and $\sigma\left(\gamma \bar{L}_{e} L_{\mu}\right)$ as function of the symmetry breaking scale $f$ for three values of the mass parameter $M_{L}$. The plots show that their values decrease as $f$ increases, which are in the ranges of $8.55-4.93 f b$ and $11.96-5.39 f b$, respectively, for $M_{L}=400 \mathrm{GeV}$ and $500 \mathrm{GeV} \leq f \leq 2000 \mathrm{GeV}$. If we assume that the future ILC experiment has a yearly integrated luminosity of $100 \mathrm{fb}^{-1}$, then several hundreds up to thousands of $\gamma \bar{L}_{i} L_{j}$ events will be generated per year.

In Fig.3 and Fig.4, we plot the distribution of transverse momentum of the final state $\gamma$ photon for the processes $e^{+} e^{-} \rightarrow \gamma \bar{L}_{\mu} L_{\mu}$ and $e^{+} e^{-} \rightarrow \gamma \bar{L}_{e} L_{\mu}$ respectively, for $f=500 \mathrm{GeV}$ and $1000 \mathrm{GeV}$ and three values of the mass parameter $M_{L}$. These figures illuminate 


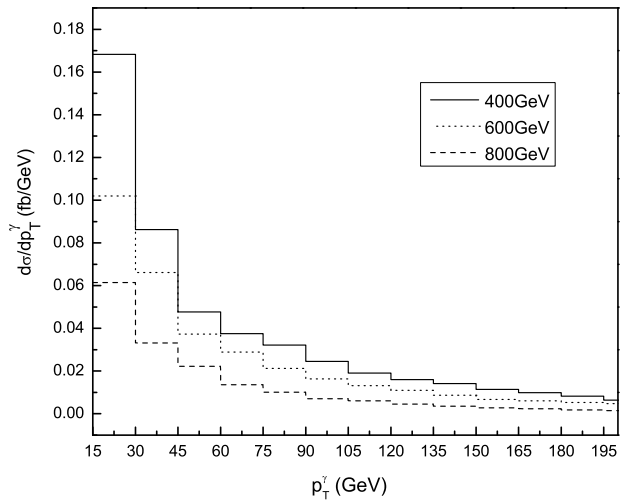

(a)

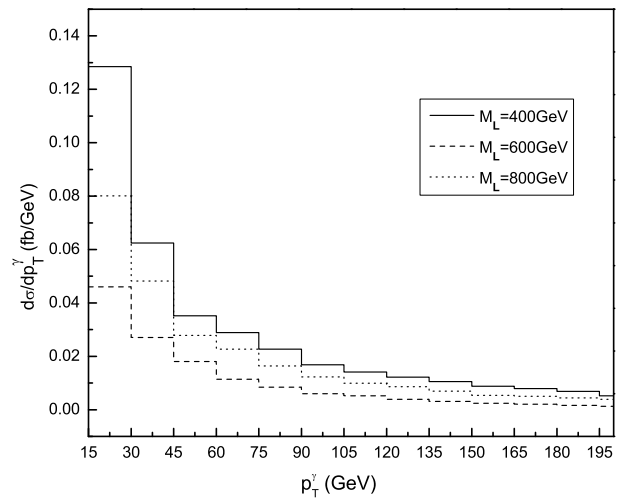

(b)

FIG. 4: The distributions of the transverse momentum of $\gamma$ photon $P_{T}^{\gamma}$ for the $e^{+} e^{-} \rightarrow \gamma \bar{L}_{\mu} L_{\mu}$ process with (a) $f=0.5 \mathrm{TeV}$, (b) $f=1 \mathrm{TeV}$ for $\sqrt{s}=2 \mathrm{TeV}$, and three values of the T-odd lepton mass $M_{L}$.

that the symmetry breaking scale $f$ and mass parameter $M_{L}$ can significantly affect the values of differential cross sections $d \sigma\left(\gamma \bar{L}_{\mu} L_{\mu}\right) / d P_{T}^{\gamma}$ and $d \sigma\left(\gamma \bar{L}_{e} L_{\mu}\right) / d P_{T}^{\gamma}$. Their values increase quickly as $P_{T}^{\gamma}$ decrease and most of the photons in the events of the processes $e^{+} e^{-} \rightarrow \gamma \bar{L}_{i} L_{j}$ are produced in the low transverse momentum range at the ILC.

To see whether the T-odd lepton $L_{i}$ can be observed at the ILC via the process $e^{+} e^{-} \rightarrow$ $\gamma \bar{L}_{i} L_{j}$, we consider the possible decay modes of the T-odd lepton $L_{i}$. From Eqs.(1) and (2), we can see that, for the Yukawa coupling constant $\kappa_{i}<0.46$, the T-odd lepton $L_{i}$ mainly decays to $B_{H} l_{i}\left(l_{i}=e, \mu\right.$ or $\left.\tau\right)$, while for $\kappa_{i}>0.46$, the T-odd leptons became heavier than the gauge bosons $W_{H}$ and $Z_{H}$ and other modes start opening up: $W_{H} l_{i}$ and $Z_{H} l_{i}[14]$. Furthermore, the mixing matrix $V_{H l}$ allows the $\mathrm{FC}$ decay $L_{i} \rightarrow B_{H} l_{j}$ with $i$ different from $j$. The partial decay width can be written in an unified manner as :

$$
\Gamma\left(L \rightarrow l V_{H}\right)=\frac{M_{L}^{3} g_{L}^{2}}{96 \pi M_{V_{H}}}\left\{x^{2}\left(1-2 x^{2}+y^{2}\right)+\left(1-y^{2}\right)^{2}\right\} \lambda^{\frac{1}{2}}\left(1, x^{2}, y^{2}\right),
$$

with $x=M_{V_{H}} / M_{L}, y=M_{l} / M_{L}$, and $\lambda(x, y, z)=x^{2}+y^{2}+z^{2}-2 x y-2 x z-2 y z$, in which $M_{V_{H}}$ is the mass of the T-odd gauge boson. $g_{L}$ represents the coupling constant of the T-odd lepton $L$ to the T-odd gauge boson $V_{H}$ and the ordinary lepton $l$. For $M_{L}<M_{Z_{H}} \simeq M_{W_{H}}$, the possible decay channels of the T-odd lepton $L_{i}$ are $L_{i} \rightarrow l_{j} B_{H}$ 
$\left(l_{j}=e, \mu\right.$ or $\left.\tau\right)$. For $f=1000 \mathrm{GeV}$ and three values of the T-odd lepton mass $M_{L}=$ $400 \mathrm{GeV}, 600 \mathrm{GeV}$, and $800 \mathrm{GeV}$, the values of the total width for the decay channels $L_{e} \rightarrow l_{i} B_{H}$ are $1.94 \mathrm{GeV}, 6.87 \mathrm{GeV}$, and $16.42 \mathrm{GeV}$, respectively. Similarly, for the decay channel $L_{\mu} \rightarrow l_{i} B_{H}$, the values of the total width are $1.95 \mathrm{GeV}, 6.91 \mathrm{GeV}$, and $16.52 \mathrm{GeV}$, respectively. Furthermore, for $f=1000 \mathrm{GeV}$, the branching ratios are $\operatorname{Br}\left(L_{e} \rightarrow \tau B_{H}\right)=$ $3 \%, \operatorname{Br}\left(L_{e} \rightarrow \mu B_{H}\right)=30 \%$ and $\operatorname{Br}\left(L_{e} \rightarrow e B_{H}\right)=67 \%$, and $\operatorname{Br}\left(L_{\mu} \rightarrow e B_{H}\right)=24.7 \%$, $\operatorname{Br}\left(L_{\mu} \rightarrow \mu B_{H}\right)=26.7 \%$ and $\operatorname{Br}\left(L_{\mu} \rightarrow \tau B_{H}\right)=48.6 \%$.

The new gauge boson $B_{H}$ which is the lightest T-odd particle can be seen as an attractive dark matter candidate [16]. The decay modes of the T-odd lepton $L_{i}$ are $L_{i} \rightarrow$ $l_{j} B_{H}\left(l_{j}=e, \mu\right.$ or $\left.\tau\right)$. Then the possible signatures of the process $e^{+} e^{-} \rightarrow \gamma \bar{L}_{e} L_{\mu}$ are the lepton flavor conservation final states $\gamma \bar{e} e+E_{T}, \gamma \bar{\mu} \mu+E_{T}$ and $\gamma \bar{\tau} \tau+E_{T}$, the lepton flavor violation final states $\gamma \bar{e} \tau+E_{T}, \gamma \bar{e} \mu+E_{T}$ and $\gamma \bar{\mu} \tau+E_{T}$. For the lepton flavor conservation signals, the intrinsic SM backgrounds mainly come from the processes $e^{+} e^{-} \rightarrow \gamma W^{+} W^{-}$ [21] with the $S M$ gauge bosons $W^{ \pm}$decay leptonically, $W^{ \pm} \rightarrow l \nu$, and the process $e^{+} e^{-} \rightarrow$ $\gamma Z Z$ [21] for one gauge boson $Z$ decaying to $\bar{l} l$ and another decaying to $\nu \bar{\nu}$. While for the lepton flavor violation signals, the main SM backgrounds come from the process $e^{+} e^{-} \rightarrow$ $\gamma W^{+} W^{-}$for all of the SM gauge bosons $W^{ \pm}$leptonic decay. For the T-odd leptons $L_{e}$ and $L_{\mu}$, the decay channels with the largest branching rations are $L_{e} \rightarrow e B_{H}$ and $L_{\mu} \rightarrow \tau B_{H}$, respectively, so we will focus on these two decay modes in our following discussions. The leading SM backgrounds of the largest production rate signals $\gamma \bar{e} \tau+E_{T}$ come from the SM process $e^{+} e^{-} \rightarrow \gamma W^{+} W^{-} \rightarrow \gamma \bar{e} \tau \nu_{e} \bar{\nu}_{\tau}$. Using the results of the branching ratios $\operatorname{Br}\left(W^{+} \rightarrow \bar{e} \nu_{e}\right)$ and $\operatorname{Br}\left(W^{-} \rightarrow \tau \bar{\nu}_{\tau}\right)$ in [20], we recalculate the cross section of the SM process $e^{+} e^{-} \rightarrow \gamma W^{+} W^{-} \rightarrow \gamma \bar{e} \tau \nu_{e} \bar{\nu}_{\tau}$, which is about $0.346 \mathrm{fb}$ at the ILC experiment with $\sqrt{s}=2 \mathrm{TeV}$. However, the cross section of the signal $\gamma \bar{e} \tau+E_{T}$ are large than $1.16 \mathrm{fb}$ in most range of the parameter space of the LHT model. Then the production rate of the intrinsic SM backgrounds is smaller than that generated by the process $e^{+} e^{-} \rightarrow \gamma \bar{L}_{e} L_{\mu}$. So, the distinct signal $\gamma \bar{e} \tau+E_{T}$ should be easily separated from the SM backgrounds. The possible signatures of the process $e^{+} e^{-} \rightarrow \gamma \bar{L}_{\mu} L_{\mu}$ are same as those of the process $e^{+} e^{-} \rightarrow \gamma \bar{L}_{e} L_{\mu}$, but, the production rates of the signatures are different for these two processes. For the process $e^{+} e^{-} \rightarrow \gamma \bar{L}_{\mu} L_{\mu}$, the signal with largest production rate is $\gamma \bar{\tau} \tau$ plus large missing energy $E_{T}, \gamma \bar{\tau} \tau+E_{T}$. Its intrinsic SM backgrounds mainly come 
from the processes $e^{+} e^{-} \rightarrow \gamma W^{+} W^{-} \rightarrow \gamma \tau \bar{\tau} \nu_{\tau} \bar{\nu}_{\tau}$ and $e^{+} e^{-} \rightarrow \gamma Z Z \rightarrow \gamma \tau \bar{\tau} \nu \bar{\nu}$ with $\nu=\nu_{e}, \nu_{\mu}, \nu_{\tau}$. At the ILC experiment with $\sqrt{s}=2 \mathrm{TeV}$, we recalculate their cross sections and find that their values are about $0.362 f b$ and $0.0094 f b$, respectively. However, the cross section of the signal $\gamma \bar{\tau} \tau+E_{T}$ are larger than $0.682 \mathrm{fb}$ in most of the parameter space of the LHT model. Thus, it may be possible to extract the signals from the backgrounds in the reasonable parameters space of the LHT model.

From the above discussions, we can see that, considering the $\mathrm{FC}$ decay $L_{i} \rightarrow B_{H} l_{j}(i \neq$ $j)$, both of the processes $e^{+} e^{-} \rightarrow \gamma \bar{L}_{e} L_{\mu}$ and $e^{+} e^{-} \rightarrow \gamma \bar{L}_{\mu} L_{\mu}$ can give rise to the signals $\gamma \bar{e} \tau+E_{T}$ and $\gamma \bar{\tau} \tau+E_{T}$. Thus, the FC decay $L_{i} \rightarrow B_{H} l_{j}(i \neq j)$ generates some interplay between $\gamma \bar{L}_{e} L_{\mu}$ and $\gamma \bar{L}_{\mu} L_{\mu}$ intermediate states. This interference effect enhances the observability of the signals and further strengthens our physical conclusions. It should be noted that this conclusion also apply other signals generated by he processes $e^{+} e^{-} \rightarrow$ $\gamma \bar{L}_{e} L_{\mu}$ and $e^{+} e^{-} \rightarrow \gamma \bar{L}_{\mu} L_{\mu}$.

\section{THE PROCESS $e^{+} e^{-} \rightarrow Z \bar{L}_{i} L_{j}$}

The T-odd leptons can also be produced in association with a gauge boson $Z$ at the ILC as shown in Fig.1. Based on the Feynman rules of the T-odd leptons written as above, the invariant production amplitude of the process $e^{+}\left(p_{1}\right) e^{-}\left(p_{2}\right) \rightarrow Z\left(p_{5}\right) \bar{L}_{i}\left(p_{4}\right) L_{j}\left(p_{3}\right)$ can be written as

$$
\begin{aligned}
\mathcal{M}_{2}= & M_{a}^{\gamma Z}+M_{a}^{Z Z}+M_{b}^{\gamma Z}+M_{b}^{Z Z}+M_{c}^{\gamma Z}+M_{c}^{Z Z}+M_{d}^{\gamma Z}+M_{d}^{Z Z}+M_{e}^{B_{H} Z} \\
& +M_{e}^{Z_{H} Z}+M_{f}^{B_{H} Z}+M_{f}^{Z_{H} Z}+M_{g}^{B_{H} Z}+M_{g}^{Z_{H} Z}+M_{h}^{B_{H} Z}+M_{h}^{Z_{H} Z}
\end{aligned}
$$

with

$$
\begin{aligned}
M_{a}^{\gamma Z}= & \frac{-i e^{3}}{S_{W} C_{W}}\left(-\frac{1}{2}+S_{W}^{2}\right) G\left(p_{1}+p_{2}, 0\right) G\left(p_{4}+p_{5}, M_{L}\right) \bar{v}\left(p_{1}\right) \gamma^{\mu} u\left(p_{2}\right) \bar{u}\left(p_{3}\right) \gamma_{\mu} \\
& \times\left[-\left(\not p_{4}+\not p_{5}\right)+M_{L}\right] \notin\left(p_{5}\right) v\left(p_{4}\right), \\
M_{a}^{Z Z}= & \frac{-i e^{3}}{S_{W}^{3} C_{W}^{3}}\left(-\frac{1}{2}+S_{W}^{2}\right)^{2} G\left(p_{1}+p_{2}, M_{Z}\right) G\left(p_{4}+p_{5}, M_{L}\right) \bar{v}\left(p_{1}\right) \gamma^{\mu} \\
& \times\left[\left(-\frac{1}{2}+S_{W}^{2}\right) P_{L}+\left(S_{W}^{2}\right) P_{R}\right] u\left(p_{2}\right) \bar{u}\left(p_{3}\right) \gamma_{\mu}\left[-\left(\not p_{4}+\not p_{5}\right)+M_{L}\right] \notin\left(p_{5}\right) v\left(p_{4}\right),
\end{aligned}
$$




$$
\begin{aligned}
& M_{b}^{\gamma Z}=\frac{-i e^{3}}{S_{W} C_{W}}\left(-\frac{1}{2}+S_{W}^{2}\right) G\left(p_{1}+p_{2}, 0\right) G\left(p_{3}+p_{5}, M_{L}\right) \bar{v}\left(p_{1}\right) \gamma^{\mu} u\left(p_{2}\right) \bar{u}\left(p_{3}\right) \notin\left(p_{5}\right) \\
& \times\left[\not p_{3}+\not p_{5}+M_{L}\right] \gamma_{\mu} v\left(p_{4}\right) \text {, } \\
& M_{b}^{Z Z}=\frac{-i e^{3}}{S_{W}^{3} C_{W}^{3}}\left(-\frac{1}{2}+S_{W}^{2}\right)^{2} G\left(p_{1}+p_{2}, M_{Z}\right) G\left(p_{3}+p_{5}, M_{L}\right) \bar{v}\left(p_{1}\right) \gamma^{\mu}\left[\left(-\frac{1}{2}+S_{W}^{2}\right) P_{L}\right. \\
& \left.+\left(S_{W}^{2}\right) P_{R}\right] u\left(p_{2}\right) \bar{u}\left(p_{3}\right) \notin\left(p_{5}\right)\left[\not p_{3}+\not p_{5}+M_{L}\right] \gamma_{\mu} v\left(p_{4}\right) \\
& M_{c}^{\gamma Z}=\frac{-i e^{3}}{S_{W} C_{W}} G\left(p_{1}-p_{5}, M_{e}\right) G\left(p_{3}+p_{4}, 0\right) \bar{v}\left(p_{1}\right) \notin\left(p_{5}\right)\left[\left(-\frac{1}{2}+S_{W}^{2}\right) P_{L}+\left(S_{W}^{2}\right) P_{R}\right] \\
& \times\left[-\left(\not p_{1}-\not p_{5}\right)+M_{e}\right] \gamma^{\mu} u\left(p_{2}\right) \bar{u}\left(p_{3}\right) \gamma_{\mu} v\left(p_{4}\right) \\
& M_{c}^{Z Z}=\frac{-i e^{3}}{S_{W}^{3} C_{W}^{3}}\left(-\frac{1}{2}+S_{W}^{2}\right) G\left(p_{1}-p_{5}, M_{e}\right) G\left(p_{3}+p_{4}, M_{Z}\right) \bar{v}\left(p_{1}\right) \notin\left(p_{5}\right)\left[\left(-\frac{1}{2}+S_{W}^{2}\right) P_{L}+\right. \\
& \left.\left(S_{W}^{2}\right) P_{R}\right]\left[-\left(\not p_{1}-\not p_{5}\right)+M_{e}\right] \gamma^{\mu}\left[\left(-\frac{1}{2}+S_{W}^{2}\right) P_{L}+\left(S_{W}^{2}\right) P_{R}\right] u\left(p_{2}\right) \bar{u}\left(p_{3}\right) \gamma_{\mu} v\left(p_{4}\right) \\
& M_{d}^{\gamma Z}=\frac{-i e^{3}}{S_{W} C_{W}} G\left(p_{2}-p_{5}, M_{e}\right) G\left(p_{3}+p_{4}, 0\right) \bar{v}\left(p_{1}\right) \gamma^{\mu}\left[\left(\not p_{2}-\not p_{5}\right)+M_{e}\right] \notin\left(p_{5}\right)\left[\left(-\frac{1}{2}+S_{W}^{2}\right) P_{L}\right. \\
& \left.+\left(S_{W}^{2}\right) P_{R}\right] u\left(p_{2}\right) \bar{u}\left(p_{3}\right) \gamma_{\mu} v\left(p_{4}\right), \\
& M_{d}^{Z Z}=\frac{-i e^{3}}{S_{W}^{3} C_{W}^{3}} G\left(p_{2}-p_{5}, M_{e}\right) G\left(p_{3}+p_{4}, M_{Z}\right)\left(-\frac{1}{2}+S_{W}^{2}\right) \bar{v}\left(p_{1}\right) \gamma^{\mu}\left[\left(-\frac{1}{2}+S_{W}^{2}\right) P_{L}+S_{W}^{2} P_{R}\right] \\
& {\left[\left(\not p_{2}-\not p_{5}\right)+M_{e}\right] \notin\left(p_{5}\right)\left[\left(-\frac{1}{2}+S_{W}^{2}\right) P_{L}+\left(S_{W}^{2}\right) P_{R}\right] u\left(p_{2}\right) \bar{u}\left(p_{3}\right) \gamma_{\mu} v\left(p_{4}\right)} \\
& M_{e}^{B_{H} Z}=\frac{-i e^{3}}{S_{W} C_{W}^{3}}\left(-\frac{1}{2}+S_{W}^{2}\right)\left[\frac{1}{10}+\frac{5 C_{W}^{2}}{8\left(5 C_{W}^{2}-S_{W}^{2}\right)} \frac{\nu^{2}}{f^{2}}\right]^{2}\left(V_{H l}\right)_{i e}\left(V_{H l}\right)_{e j} \\
& \times G\left(p_{4}+p_{5}, M_{L}\right) G\left(p_{2}-p_{3}, M_{B_{H}}\right) \bar{v}\left(p_{1}\right) \gamma^{\mu} P_{L}\left[-\left(\not p_{4}+\not p_{5}\right)+M_{L}\right] \\
& \left.\times \notin\left(p_{5}\right) v_{(} p_{4}\right) \bar{u}\left(p_{3}\right) \gamma_{\mu} P_{L} u\left(p_{2}\right), \\
& M_{e}^{Z_{H} Z}=\frac{-i e^{3}}{S_{W}^{3} C_{W}}\left(-\frac{1}{2}+S_{W}^{2}\right)\left[-\frac{1}{2}+\frac{S_{W}^{2}}{8\left(5 C_{W}^{2}-S_{W}^{2}\right)} \frac{\nu^{2}}{f^{2}}\right]^{2}\left(V_{H l}\right)_{i e}\left(V_{H l}\right)_{e j} \\
& \times G\left(p_{4}+p_{5}, M_{L}\right) G\left(p_{2}-p_{3}, M_{Z_{H}}\right) \bar{v}\left(p_{1}\right) \gamma^{\mu} P_{L}\left[-\left(\not p_{4}+\not p_{5}\right)+M_{L}\right] \\
& \times \notin\left(p_{5}\right) v\left(p_{4}\right) \bar{u}\left(p_{3}\right) \gamma_{\mu} P_{L} u\left(p_{2}\right), \\
& M_{f}^{B_{H} Z}=\frac{-i e^{3}}{S_{W} C_{W}^{3}}\left(-\frac{1}{2}+S_{W}^{2}\right)\left[\frac{1}{10}+\frac{5 C_{W}^{2}}{8\left(5 C_{W}^{2}-S_{W}^{2}\right)} \frac{\nu^{2}}{f^{2}}\right]^{2}\left(V_{H l}\right)_{i e}\left(V_{H l}\right)_{e j} G\left(p_{4}-p_{1}, M_{B_{H}}\right) \\
& \times G\left(p_{3}+p_{5}, M_{L}\right) \bar{v}\left(p_{1}\right) \gamma^{\mu} P_{L} v\left(p_{4}\right) \bar{u}\left(p_{3}\right) \notin\left(p_{5}\right)\left[\not p_{3}+\not p_{5}+M_{L}\right] \gamma_{\mu} P_{L} u\left(p_{2}\right),
\end{aligned}
$$




$$
\begin{aligned}
M_{f}^{Z_{H} Z}= & \frac{-i e^{3}}{S_{W}^{3} C_{W}}\left(-\frac{1}{2}+S_{W}^{2}\right)\left[-\frac{1}{2}+\frac{S_{W}^{2}}{8\left(5 C_{W}^{2}-S_{W}^{2}\right)} \frac{\nu^{2}}{f^{2}}\right]^{2}\left(V_{H l}\right)_{i e}\left(V_{H l}\right)_{e j} G\left(p_{4}-p_{1}, M_{Z_{H}}\right) \\
& \times G\left(p_{3}+p_{5}, M_{L}\right) \bar{v}\left(p_{1}\right) \gamma^{\mu} P_{L} v\left(p_{4}\right) \bar{u}\left(p_{3}\right) \notin\left(p_{5}\right)\left[\not p_{3}+\not p_{5}+M_{L}\right] \gamma_{\mu} P_{L} u\left(p_{2}\right), \\
M_{g}^{B_{H} Z}= & \frac{-i e^{3}}{S_{W} C_{W}^{3}}\left[\frac{1}{10}+\frac{5 C_{W}^{2}}{8\left(5 C_{W}^{2}-S_{W}^{2}\right)} \frac{\nu^{2}}{f^{2}}\right]^{2}\left(V_{H l}\right)_{i e}\left(V_{H l}\right)_{e j} G\left(p_{5}-p_{1}, M_{e}\right) G\left(p_{2}-p_{3}, M_{B_{H}}\right) \\
& \times \bar{v}\left(p_{1}\right) \notin\left(p_{5}\right)\left(-\frac{1}{2} P_{L}+S_{W}^{2}\right)\left[\not p_{5}-\not p_{1}+M_{e}\right] \gamma^{\mu} P_{L} v\left(p_{4}\right) \bar{u}\left(p_{3}\right) \gamma_{\mu} P_{L} u\left(p_{2}\right), \\
M_{g}^{Z_{H} Z}= & \frac{-i e^{3}}{S_{W}^{3} C_{W}}\left[-\frac{1}{2}+\frac{S_{W}^{2}}{8\left(5 C_{W}^{2}-S_{W}^{2}\right)} \frac{\nu^{2}}{f^{2}}\right]^{2}\left(V_{H l}\right)_{i e}\left(V_{H l}\right)_{e j} G\left(p_{5}-p_{1}, M_{e}\right) G\left(p_{2}-p_{3}, M_{Z_{H}}\right) \\
& \times \bar{v}\left(p_{1}\right) \notin\left(p_{5}\right)\left(-\frac{1}{2} P_{L}+S_{W}^{2}\right)\left[\not p_{5}-\not p_{1}+M_{e}\right] \gamma^{\mu} P_{L} v\left(p_{4}\right) \bar{u}\left(p_{3}\right) \gamma_{\mu} P_{L} u\left(p_{2}\right), \\
M_{h}^{B_{H} Z}= & \frac{-i e^{3}}{S_{W} C_{W}^{3}}\left[\frac{1}{10}+\frac{5 C_{W}^{2}}{8\left(5 C_{W}^{2}-S_{W}^{2}\right)} \frac{\nu^{2}}{f^{2}}\right]^{2}\left(V_{H l}\right)_{i e}\left(V_{H l}\right)_{e j} G\left(p_{4}-p_{1}, M_{B_{H}}\right) G\left(p_{2}-p_{5}, M_{e}\right) \\
& \times \bar{v}\left(p_{1}\right) \gamma^{\mu} P_{L} v\left(p_{4}\right) \bar{u}\left(p_{3}\right) \gamma_{\mu} P_{L}\left[\not p_{2}-\not p_{5}+M_{e}\right] \notin\left(p_{5}\right)\left(-\frac{1}{2} P_{L}+S_{W}^{2}\right) u\left(p_{2}\right), \\
M_{h}^{Z_{H} Z}= & \frac{-i e^{3}}{S_{W}^{3} C_{W}}\left[-\frac{1}{2}+\frac{S_{W}^{2}}{8\left(5 C_{W}^{2}-S_{W}^{2}\right)} \frac{\nu^{2}}{f^{2}}\right]^{2}\left(V_{H l}\right)_{i e}\left(V_{H l}\right)_{e j} G\left(p_{4}-p_{1}, M_{Z_{H}}\right) G\left(p_{2}-p_{5}, M_{e}\right) \\
& \times \bar{v}\left(p_{1}\right) \gamma^{\mu} P_{L} v\left(p_{4}\right) \bar{u}\left(p_{3}\right) \gamma_{\mu} P_{L}\left[\not p_{2}-\not p_{5}+M_{e}\right] \notin\left(p_{5}\right)\left(-\frac{1}{2} P_{L}+S_{W}^{2}\right) u\left(p_{2}\right) .
\end{aligned}
$$

Similar with above, we can give the numerical results about the process $e^{+} e^{-} \rightarrow Z \bar{L}_{i} L_{j}$, which are summarized in Fig.5. We plot the cross sections of the processes $e^{+} e^{-} \rightarrow Z \bar{L}_{e} L_{\mu}$ and $e^{+} e^{-} \rightarrow Z \bar{L}_{\mu} L_{\mu}$ as functions of the symmetry breaking scale $f$ for three values of the mirror lepton mass $M_{L}$ in Fig.5. One can see that the cross sections $\sigma\left(Z \bar{L}_{e} L_{\mu}\right)$ and $\sigma\left(Z \bar{L}_{\mu} L_{\mu}\right)$ fall sharply as $f$ increases for fixed T-odd lepton mass $M_{L}$ and their values are also sensitive to the mass of the T-odd leptons, which is similar with those of the processes $e^{+} e^{-} \rightarrow \gamma \bar{L}_{e} L_{\mu}$ and $e^{+} e^{-} \rightarrow \gamma \bar{L}_{\mu} L_{\mu}$. This is because the phase space is depressed strongly by large final states and the coupling are related to the factor $\nu^{2} / f^{2}$. The cross sections $\sigma\left(Z \bar{L}_{e} L_{\mu}\right)$ and $\sigma\left(Z \bar{L}_{\mu} L_{\mu}\right)$ are smaller than those of the processes $e^{+} e^{-} \rightarrow \gamma \bar{L}_{e} L_{\mu}$ and $e^{+} e^{-} \rightarrow \gamma \bar{L}_{\mu} L_{\mu}$, respectively. For $f=500-2000 \mathrm{GeV}$ and $M_{L}=400-800 \mathrm{GeV}$, the value of cross section $\sigma\left(Z \bar{L}_{e} L_{\mu}\right)\left[\sigma\left(Z \bar{L}_{\mu} L_{\mu}\right)\right]$ is in the range of $1.62-0.187 f b[1.21-0.171 f b]$. The distributions of the transverse momentum of the gauge boson $Z$ are depicted in Fig.6 


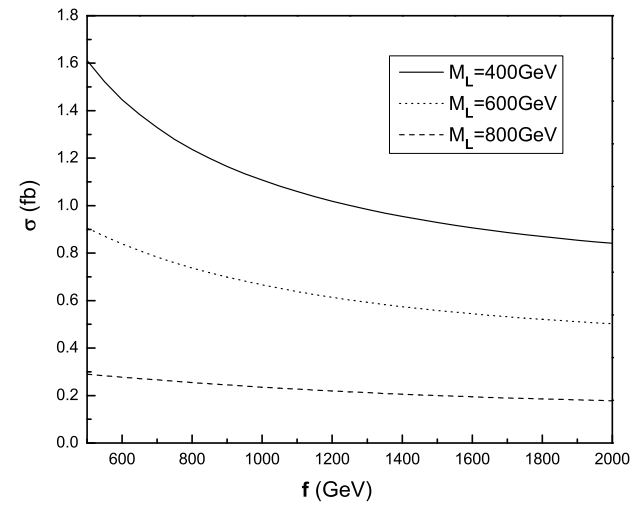

(a)

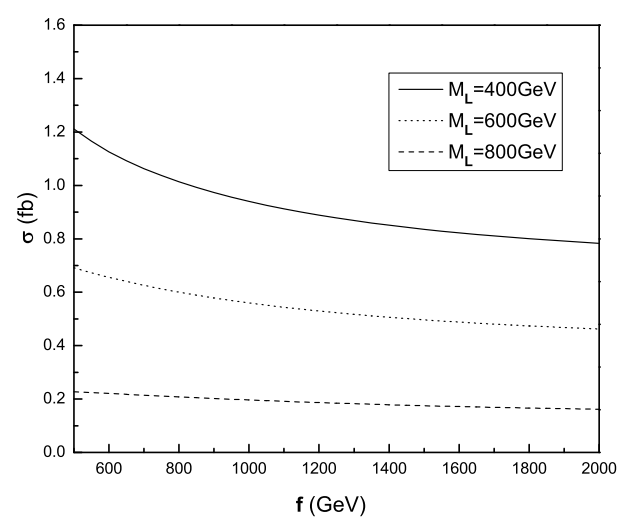

(b)

FIG. 5: The production cross sections (a) $\sigma\left(Z \bar{L}_{e} L_{\mu}\right)$ and (b) $\sigma\left(Z \bar{L}_{\mu} L_{\mu}\right)$ as function of the scale parameter $f$ for $\sqrt{s}=2 T e V$ and three values of the T-odd lepton mass $M_{L}$.

and Fig.7 corresponding to $f=0.5 \mathrm{TeV}$ and $1 \mathrm{TeV}$, respectively. From these two figures we can see that there exist peaks at different conditions, there are significant regions of $P_{T}^{Z}$ with differential values of the symmetry breaking scale $f$ and the mirror lepton mass $M_{L}$. The larger values of the symmetry breaking scale $f$ and the mirror lepton mass $M_{L}$ can significantly suppress the cross sections $d \sigma\left(Z \bar{L}_{\mu} L_{\mu}\right) / d P_{T}^{Z}$ and $d \sigma\left(Z \bar{L}_{e} L_{\mu}\right) / d P_{T}^{Z}$. Obviously, for $f \geq 1 \mathrm{TeV}$ and $M_{L} \geq 800 \mathrm{GeV}$, the values of cross sections are quite small.

If we assume the final state $Z$ decaying to $\bar{l} l$, the possible signatures of the process $e^{+} e^{-} \rightarrow Z \bar{L}_{e} L_{\mu}$ are the lepton flavor conservation final states $\bar{l} l \bar{e} e+E_{T}, \bar{l} l \bar{\mu} \mu+E_{T}$ and $\bar{l} l \bar{\tau} \tau+E_{T}$, and the lepton flavor violation final states $\bar{l} l \bar{e} \tau+E_{T}, \bar{l} l \bar{e} \mu+E_{T}$ and $\bar{l} l \bar{\mu} \tau+E_{T}$. For the lepton flavor conservation signals, the intrinsic SM backgrounds mainly come from the processes $e^{+} e^{-} \rightarrow Z W^{+} W^{-}[22]$ with the gauge bosons $W^{ \pm}$decay leptonically, $W^{ \pm} \rightarrow l \nu$, and the process $e^{+} e^{-} \rightarrow Z Z W^{+} W^{-}$for one gauge boson $Z$ decaying to $\bar{l} l$ and other decaying to $\nu \bar{\nu}$.

While for the lepton flavor violation signals, the main SM backgrounds come from the processes $e^{+} e^{-} \rightarrow Z W^{+} W^{-}$with the gauge boson $Z$ decaying to $\bar{l} l$ and the gauge bosons $W^{ \pm}$leptonic decay, and the process $e^{+} e^{-} \rightarrow Z Z W^{+} W^{-}$for all of the gauge bosons $W^{ \pm}$ 


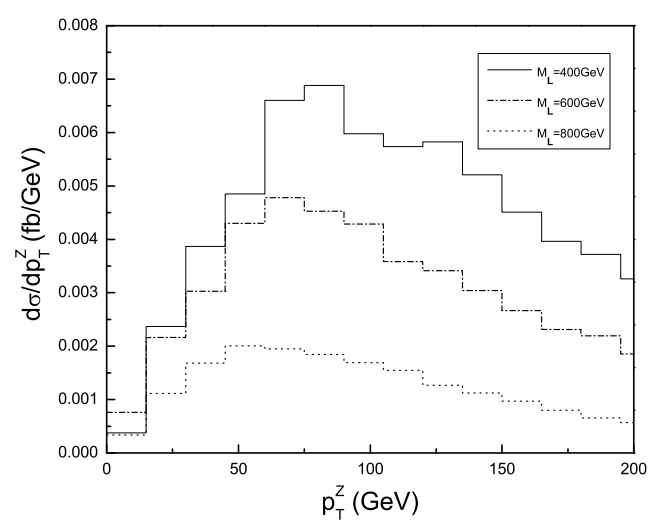

(a)

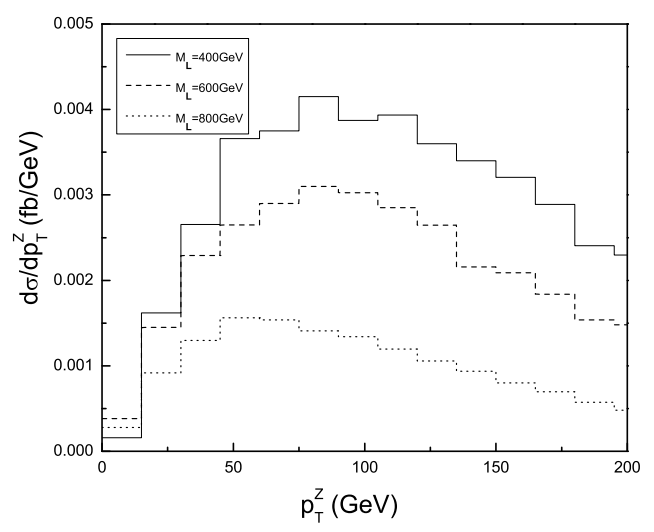

(b)

FIG. 6: The distributions of the transverse momentum of $Z$ boson $P_{T}^{Z}$ for the $e^{+} e^{-} \rightarrow Z \bar{L}_{e} L_{\mu}$ process with $f=0.5 \mathrm{TeV}$ (a) and $f=1 \mathrm{TeV}$ (b) for $\sqrt{s}=2 \mathrm{TeV}$, and three values of the T-odd lepton mass $M_{L}$.

leptonic decay, and one gauge boson $Z$ decaying to $\bar{l}$ another decaying to $\nu \bar{\nu}$. From discussions given by section II, it is obviously that, the largest production rate signal of the process $e^{+} e^{-} \rightarrow Z \bar{L}_{e} L_{\mu}$ is $\bar{l} l \bar{e} \tau+E_{T}$. The leading SM backgrounds of this kind of signal come from the SM processes $e^{+} e^{-} \rightarrow Z W^{+} W^{-} \rightarrow \bar{l} l \bar{e} \tau \nu_{e} \bar{\nu}_{\tau}$, and $e^{+} e^{-} \rightarrow Z Z W^{+} W^{-} \rightarrow$ $\bar{l} l \bar{e} \tau \nu_{e} \bar{\nu}_{\tau} \nu \bar{\nu}\left(\nu=\nu_{e}, \nu_{\mu}, \nu_{\tau}\right)$. Our numerical results indicate that, in wide range of the parameter space of the LHT model, the value of the statistical significance $S / \sqrt{B}$ is larger than 5. In our numerical estimation, we have taken the integrated luminosity $£=100 \mathrm{fb}^{-1}$ and $\sqrt{S}=2 \mathrm{TeV}$. Thus, it may be possible to extract the signals from the backgrounds in the reasonable parameter space of the LHT model. The possible signatures of the process $e^{+} e^{-} \rightarrow Z \bar{L}_{\mu} L_{\mu}$ are same as that of the process $e^{+} e^{-} \rightarrow Z \bar{L}_{e} L_{\mu}$, but, the production rates are different from those generated by the process $e^{+} e^{-} \rightarrow Z \bar{L}_{e} L_{\mu}$. For the process $e^{+} e^{-} \rightarrow Z \bar{L}_{\mu} L_{\mu}$, the signal with largest production rate is the same-flavor opposite-sign pair leptons $\bar{l} l \bar{\tau} \tau$ plus large missing energy $E_{T}$; i.e. $\bar{l} l \bar{\tau} \tau+E_{T}$. The SM backgrounds mainly come from the $e^{+} e^{-} \rightarrow Z W^{+} W^{-} \rightarrow \bar{l} l \tau \bar{\tau} \nu_{\tau} \bar{\nu}_{\tau}$ and $e^{+} e^{-} \rightarrow Z Z W^{+} W^{-} \rightarrow \bar{l} l \tau \bar{\tau} \nu_{\tau} \bar{\nu}_{\tau} \nu \bar{\nu}$ $\left(\nu=\nu_{e}, \nu_{\mu}, \nu_{\tau}\right)$. Our numerical results indicate that, the value of the statistical significance $S / \sqrt{B}$ is larger than 3 in wide range of the parameter space of the LHT model. Thus, as 


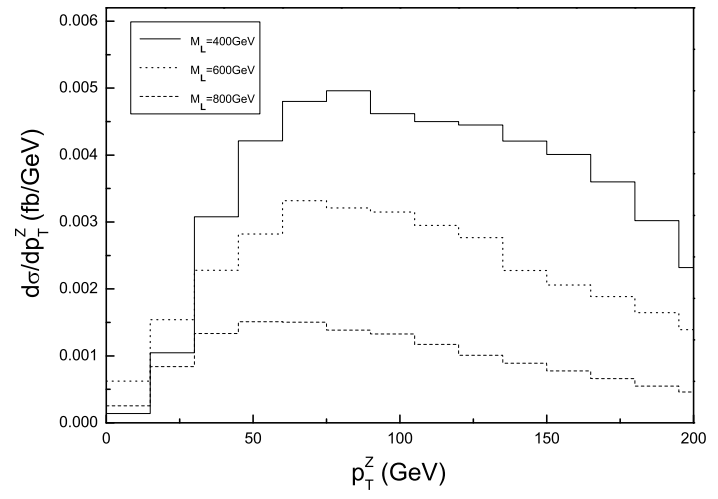

(a)

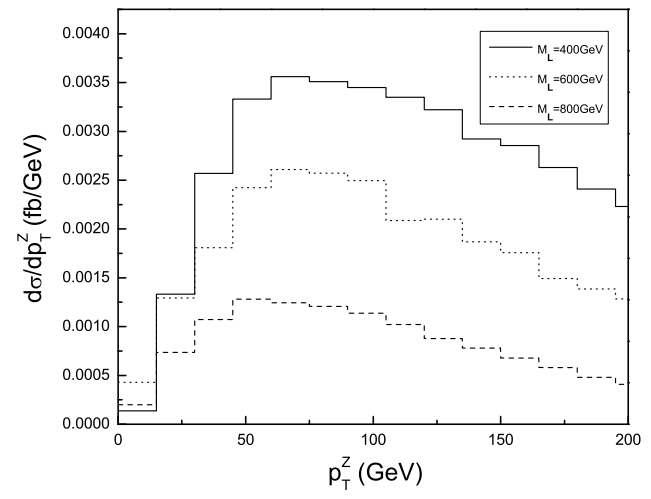

(b)

FIG. 7: The distributions of the transverse momentum of $Z$ boson $P_{T}^{Z}$ for the $e^{+} e^{-} \rightarrow Z \bar{L}_{\mu} L_{\mu}$ process with $f=0.5 \mathrm{TeV}$ (a) and $f=1 \mathrm{TeV}$ (b) for $\sqrt{s}=2 \mathrm{TeV}$, and three values of the T-odd lepton mass $M_{L}$.

long as the T-odd leptons are not too heavy, its possible signals might be detected via the processes $e^{+} e^{-} \rightarrow Z \bar{L}_{i} L_{j}$ in the future ILC experiments.

Certainly, the SM backgrounds must be further studied, detailed confirmation of the observability of the signals generated by the process $e^{+} e^{-} \rightarrow V \bar{L}_{i} L_{j}$ would require more Monte Carlo simulations of the signals and backgrounds, which is beyond the scope of this paper.

\section{CONCLUSIONS}

The LHT model is one of the attractive little Higgs models, which not only is consistent with EW precision tests but also provides a possible dark matter candidate. The heavy T-odd fermions (mirror leptons and mirror quarks) are introduced to implement T-parity in the fermion sector of the model. These new heavy fermions might produce the observability signatures in future high energy collider experiments.

In this paper we consider pair production of the T-odd leptons in association with a gauge boson $V(=\gamma$ or $Z)$ in the future ILC experiments. The production cross sections of these processes and their distributions of the transverse momentum are calculated. 
Our numerical results show that the cross section of the process $e^{+} e^{-} \rightarrow \gamma \bar{L}_{i} L_{j}$ is larger than that of the process $e^{+} e^{-} \rightarrow Z \bar{L}_{i} L_{j}$. For $\sqrt{s}=2 T e V, M_{L}=400-800 G e V$ and $f=500-2000 \mathrm{GeV}$, the values of the cross sections $\sigma\left(\gamma \bar{L}_{e} L_{\mu}\right)$ and $\sigma\left(\gamma \bar{L}_{\mu} L_{\mu}\right)$ are in the ranges of $11.96-2.16 \mathrm{fb}$ and $8.55-1.89 \mathrm{fb}$, while those for $\sigma\left(Z \bar{L}_{e} L_{\mu}\right)$ and $\sigma\left(Z \bar{L}_{\mu} L_{\mu}\right)$ are in the ranges of $1.62-0.187 f b$ and $1.21-0.171 f b$. We further analyze their possible signals and the corresponding SM backgrounds, also calculate the value of the statistical significance $S / \sqrt{B}$ for some processes. We find that, as long as the T-odd leptons are not too heavy, they can be copiously produced via the processes $e^{+} e^{-} \rightarrow V \bar{L}_{i} L_{j}$, and their signatures might be observed in the future ILC experiments. Thus, we expect that these production processes can be used to detect the T-odd leptons predicted by the LHT model in the future ILC experiments.

\section{Acknowledgments}

This work was supported in part by the National Natural Science Foundation of China under Grants No.10975067, the Specialized Research Fund for the Doctoral Program of Higher Education (SRFDP) (No.200801650002), the Natural Science Foundation of the Liaoning Scientific Committee (No. 201102114), and Foundation of Liaoning Educational Committee (No. LT2011015).

[1] N. Arkani-Hamed, A. G. Cohen, H. Georgi, Phys. Lett. B 513 (2001) 232;

N. Arkani-Hamed et al., JHEP 0208 (2002) 020;

I. Low, W. Skiba, D. Smith, Phys. Rev. D 66 (2002) 072001;

D. E. Kaplan, M. Schmaltz, JHEP 0310 (2003) 039.

[2] N. Arkani-Hamed, A. G. Cohen, E. Katz, A. E. Nelson, JHEP 0207 (2002) 034;

T. Han, H. E. Logan, B. McElrath, L. T. Wang, Phys. Rev. D 67 (2003) 095004;

M. Schmaltz, D. Tucker-smith, Ann. Rev. Nucl. Part. Sci. 55 (2005) 229.

[3] J. L. Hewett, F. J. Petriello, T. G. Rizzo, JHEP 0310 (2003) 062; 
M. C. Chen, S. Dawson, Phys. Rev. D 70 (2004) 015003; W. Kilian and J. Reuter, Phys. Rev. D 70 (2004)015004; T. Han, H. E. Logan, B. McElrath, L. T. Wang, Phys. Lett. B $563(2003) 191$.

[4] H. C. Cheng, I. Low, JHEP 0309 (2003) 051;

H. C. Cheng, I. Low, JHEP 0408 (2004) 061.

[5] I. Low, JHEP 0410 (2004) 067;

J. Hubisz, P. Meade, Phys. Rev. D 71 (2005) 035016.

[6] M. Blake et al., JHEP 0701, (2007) 066.

[7] J. Hubisz, P. Meade, A. Noble, M. Perelstein, JHEP 0601 (2006) 135;

A. Freitas, D. Wyler, JHEP 0611 (2006) 061;

M. Blanke et al., JHEP 0612 (2006) 003;

J. Hubisz, S. J. Lee, G. Paz, JHEP 0606 (2006) 041.

[8] M. Blanke et al., Phys. Lett. B 646 (2007) 253.

[9] Hou Hong-Sheng, Phys. Rev. D 75 (2007) 094010;

M. Blanke et al., Phys. Lett. B 657 (2007) 81;

M. Blanke et al., JHEP 0706 (2007) 082.

[10] M. Blanke et al., JHEP 0705 (2007) 013;

S. R. Choudhury et al., Phys. Rev.D 75 (2007) 055011.

[11] F. del Aguila, J. I. Illana, M. D. Jenkins, JHEP 0901 (2009) 080;

M. Blanke et al., Acta Phys. Polon. B 41 (2010) 657;

T. Goto, Y. Okada, Y. Yamamoto, Phys. Rev. D 83 (2011) 053011;

F. del Aguila, J. I. Illana, M. D. Jenkins, JHEP 1009 (2010) 040.

[12] Wei Liu, Chong-Xing Yue, Jiao Zhang, Eur. Phys. J. C 68 (2010) 197;

Chong-Xing Yue, Jin-Yan Liu, Shi-Hai Zhu, Phys. Rev. D 78 (2008) 095006.

[13] G. Weiglein et al. [LHC/LC Study Group], Phys.Rept. 426 (2006) 47.

[14] G. Cacciapaglia, A. Deandrea, S. R. Choudhury, N. Gaur, Phys. Rev. D 81 (2010) 075005;

Chong-Xing Yue, Yong-Zhi Wang, Wei Lui, Wei Ma, Europhys. Lett 86 (2009) 31002.

[15] Chong-Xing Yue, Nan Zhang, Shi-Hai Zhu, Eur. Phys. J. C 53 (2008) 215.

[16] A. Birkedal, A. Noble, M. Perelstein, A. Spary, Phys. Rev. D 74 (2006) 035002;

M. Asano et al., Phys. Rev. D 75 (2007) 063506; 
C. S. Chen, K. Cheung, T. C. Yuan, Phys. Lett. B 644 (2007) 158;

M. Perelstein, A. Spray, Phys. Rev. D 75 (2007) 083519.

[17] J. D. Bjorken, P. F. Harrison, W. G. Scott, Phys. Rev. D 74 (2006) 073012;

M. J. Baker, J. Bordes, H. M. Chan, S. T. Tsou, arXiv: 1110.3951 [hep-ph].

[18] I. Cortes-Maldonado, G. Tavares-Velasco, Int. J. Mod. Phys. A 26 (2011) 5349.

[19] K. Hagiwara, D. Zeppenfeld. Nucl. Phys. B 313 (1989) 560;

V. D. Barger, T. Han, D. Zeppenfeld, Phys. Rev. D 41 (1990) 2782.

[20] K. Nakamura et al. [Particle Data Group], J. Phys. G 37 (2010) 075021.

[21] W. J. Stirling, A. Werthenbach, Eur. Phys. J. C 14 (2000) 103.

[22] V. D. Barger, T. Han, Phys. Lett. B 212 (1988) 117;

V. D. Barger, T. Han, R. Phillips, Phys. Rev. D 39 (1989) 146;

A. Tofighi-Niaki, J. Gunion, Phys. Rev. D 39 (1989) 720. 